\title{
Root Reasons behind the Unusual Behaviors of the Earth climate thus the causes of Natural Disasters
}

\author{
Dr. Abu Mohammad Azmal Morshed \\ Department of Chemistry, Primeasia University, 9, Kemal Ataturk Avenue, Banani, Dhaka-1213.
}

\begin{abstract}
Natural disaster have a number of root causes, but today with global warming, natural disasters have as their root cause, the change in the environment triggered by increasing differences in heat and cold. Greenhouse gases increase overall temperatures and this is masked by particles that contribute to global dimming. At the local level, global dimming causes drops in temperature and in areas where this is not the major factor, temperatures increase. As the atmosphere seeks to balance extremes, violent weather phenomena that contribute to natural disasters become more frequent. The results are more floods, drying leading to fire-storms, high winds, unseasonal freezes, ice-pack break up and meltdown, super hurricanes and rising sea levels that are a significant proportion of natural disasters today. Other natural disasters like tsunamis, solar dimming, volcanoes and impacts from space are not man made at this point in history and these will be not considered here. It comes as no surprise to many people today that there are records. In this article I have pointed out the main reason why the world weather is behaving rough.
\end{abstract}

Key words: Environment, disaster, electrolyte, balances etc.

\section{Introduction:}

Climate is the long-term statistical expression of short-term weather. Climate can be defined as "expected weather". When changes in the expected weather occur, we call these climate changes. They can be defined by the differences between average weather conditions at two separate times. Climate may change in different ways, over different time scales and at different geographical scales. In recent times, scientists have become interested in global warming, due to mankind's impact on the climate system, through the enhancement of the natural greenhouse effect. In this article I have pointed out the main reason behind the rapid weather change around the world. Up to now the overall state of the global climate is determined by the amount of energy stored by the climate system, and in particular the balance between energy the earth receives from the Sun and the energy which the earth releases back to space, called the global energy balance. How this energy balance is regulated depends upon the flows of energy within the global climate system. Major causes of climate change involve any process that can alter the global energy balance, and the energy flows within the climate system. Causes of climate change include changes in the earth's orbit around the sun, changes in the amount of energy coming from the Sun, changes in ocean circulation or changes in the composition of the atmosphere. By contrast, the movement of continents around the world over hundreds of millions of years can also affect global climate, but only over these much longer time scales. This is not the full picture of the roots of natural disasters. Earthquake connections to sea level rise as caused by human activity are controversial. However, there is some evidence that human activity elsewhere is linked to low level earthquakes. Other natural disasters like tsunamis, solar dimming, volcanoes and impacts from space are not man made at this point in history and these will be not considered here.

It comes as no surprise to many people today that there are records being broken everywhere as far as weather anomalies are concerned and one of these is flooding. Global warming is seen as a contributor to increasing amounts of moisture in the air as the water on ocean surfaces evaporates more readily. As this blows over land, it condenses in regions that are naturally susceptible to rainfall such as near mountains. These areas see a lot of flooding. Also, more moisture translates into more snow and in the spring it means more run off from melting and thus more flooding. In the fading stages of the 2009 winter, a large accumulation of snow meant a large risk of flood along the Red River flood basin. In fact, the water levels were higher than anything recorded in the last 112 years. It was in Asia Minor that experienced massive flash floods unlike anything seen in recent memory. These have been tentatively linked to global warming caused by human injection of greenhouse gasses into the atmosphere from industrial processes and engines of all sorts.

\section{Discussion:}

Throughout the Earth's history climate has fluctuated between periods of relative warmth and relative cold. Paleoclimatology is the study of climate and climate change prior to the period of direct measurements. Direct records of temperature and other climatic elements span only a tiny fraction of the Earth's climatic history, and so provide an inadequate perspective on climatic change and the evolution of the climate today and 
in the future. A longer perspective on climate variability can be obtained by the study of natural phenomena which are climate-dependent.

\section{Reason Identification:}

The root reasons for the global climate change has been described in many ways, showing many major and minor factors responsible behind. Fact whatever may behind result is very alarming for the living beings and to the overall existence of the universe. Counties such as Bangladesh, Maldives, Part of India and many other costal countries whose land height is very little difference with the sea level and now it is well documented that many part of these lower height countries will go under sea level. It has been the fact that global climate change is responsible for the unusual behavior of the earth i,e., Earth quick, lands light, land fall, Tsunamis, depression around the world, Cyclones, Smog, acid rain, Heavy rainfall, rain less over year to year and heavy snowfall years in many areas of the world.

It could be correlate the introduce of many new diseases caused by the introduction of new virus, bacteria's or even by new cancer cells in the living beings due to the change of world environment. If these happenings continue over year after year then there will be introduce of many other natural calamities which will turn to must causing alarming devastating epidemic for the existence of living beings in this universe. I propose here about the significant reason that could be said as the root cause of the world environmental change which is effecting on the suitable conditions of the living beings existence and about the possible recovery from the alarming situation. I strongly propose thus we can save the living beings suitable existence in the world if we can resolve the problem. If we mention the golden period of the earth condition for living beings than it will be hardly few thousand years back to tail of nineteen century. Reviewing the world environment condition of the mentioned age it is found that, this period was backboned on natural living system (nonscientific) or may be just the beginning of the scientific ages.

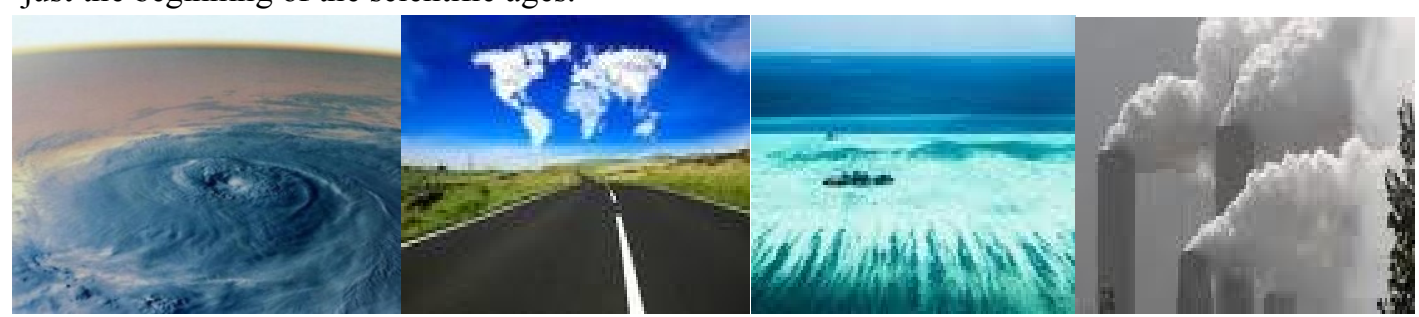

Figure 1: Industrial processes, coal and fossil fuel burning and deforestation all contribute to global dimming and wild weather.

\section{Root Reason behind the World Weather Change:}

If we look around earth's environment along with the space we find the world is surrounded by three main atmospheric layers and the function /usefulness of these layers are to act as filter to protect the earth from many hazardous rays of the sun and also from the other disaster elements of the space thus to make the earth suitable for living beings. I would like to introduce here the main concern about the atmospheric layer is about its dual activities first one as the filter of the earth and the second one is the atmospheric layers act as the shed or boundary to keep the earth's environmental condition suitable for living beings there. The layers also restrict the mixing of the earth environment with the more hazardous space environment.

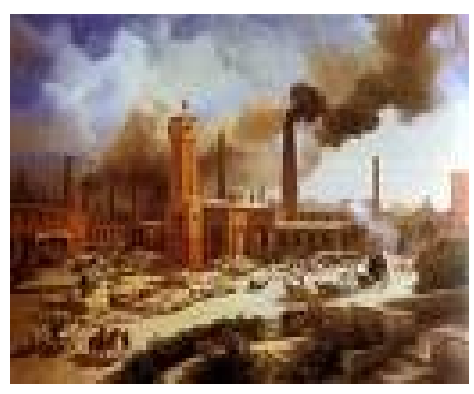

If we look to the components and the composition of the earth surface we find more than two third of the total earth's area is occupied with liquid component mainly with water that is also main component of living cells constituents. Among the existing total amount of liquid on the earth surface most amount are saline water in the oceans, seas and in the gulf. Rest water is sweet but having the same properties of polar nature in the sources of rivers, cannels and in the ponds. My main attention is on the fact of salinity of the water sources on the earth surface and their role on the total earth environment.

In the saline water, metal ion is the main constituent's components that are forming ionic/polar bonded compounds with halogens. The situation is that, most of the existing liquids on the earth's surroundings are in polar form which is in term in the form of partially ionic condition. If we deepened our concern the earth surface is accompanied with abundant of ionic (polar) components and how the situation was made stable on the earth surface that was suitable for living beings existence. 
The fact of the gradually losing electrolytic balance can be pointed out as the use of the electrolytes by living beings over million/billion years through the total mass of the earth. Besides, the sweet water percent is also adding for the last few decades by melting snow and freezing water from the storage of the end corner of the earth and also from the many points of the earth due to the raise of the overall temperature of the earth surface.

All living beings collect their required energy to lead their mode of life using the available sources related with the earth existing balancing system. By the way the earth is losing (directly or indirectly) the balancing properties from the existing suitable conditions for the living beings on the earth surface. Inventions of various new scientific equipments using the available sources from the earth, causing the loss of the total electrolytic balancing system throughout the earth surface. Moreover the radioactivity of many elements both natural and artificially causes the serious damage to the total electrolytic balancing systems across the earth environment. Even the productions of various new living organs inside the living being using the earth sources causing the deficiency in the total available balancing systems across the earth. These are all spontaneous process and are increasing more rapidly day to day with the modernization which is much alarming to the loosing of total balancing system across the earth. From these above facts it may mentioned that the world existence is under threat.

\section{Suggestions for recovery:}

Answer may come by the way that there are must an exact electrostatic balance between the aqueous electrolytes (sea water) and the existing all other components (ionic, nonionic, covalent, and neutral) inside the atmospheric boundaries along with the components of the mines within the total mass of the globe. There may have other facts like attraction/interaction force i.e., Van deer Waal's attraction forces, dipole- dipole interactions (associate the molecular level) between the abundant polar solvent (water) around the earth's surface and between other components within the range of the earth. If we come to the main concern of the global climate change relating the change of the quality and quantity of the sea (saline) water which is effecting on the total electrolytic purity/quantity, causing the electrolytic imbalance inside the atmospheric boundaries around the total mass area of the earth. The fact of lost electrolytic balance is the main reasons for the unusual behavior of the climate around the globe. This reason also may responsible for unique happenings in the space near to the earth.

\section{Conclusion:}

This has reached up to sky scraping success with many inventions. So there is nothing to be worried about the climate changing effect on the world environment. We can find out the root cause of the happenings. According to my assumption, the electrolytic imbalances between existing electrolytes in the sea water and with the other electrolytic earth components which is causing the rapid climate change across the world that is causing the unusual behaviors of the earth climate across our loving suitable earth surface. This assumption can be proven by making artificial pilot earth environment to observe the effects of the electrolytic imbalances on the living beings and on the environment. Thus we can get near to the door of the solution to get rid of the world environmental disasters.

References: Assumptions \& web sites. 